\title{
DYNAMIC ANALYSIS OF GROUPED PILE FOUNDATION IN LAYERED SOILS
}

\author{
By Hirokazu TAKEMIYA* and Yasuyuki YUKAWA**
}

\begin{abstract}
The dynamics of a grouped pile foundation in layered solis are investigated from the distributed parameter system modeling with a due consideration of the pile-soil-pile interaction. The substructure concept is applied to evaluate dynamic soil stiffness. The soil-pile coupling motion is solved for the base input through the eigenvalue decomposition and the transfer matrix method, deriving the pile head impedance function and the associated driving force (effective input). An efficient simplified mehod is also developed based on the "ring-pile" idea. From a numerical example, special remarks are given on the dynamic pile grouping effect.
\end{abstract}

\section{INTRODUCTION}

Pile foundations have been used at deep soft soil deposits to support superstructures safely. The dynamics of such pile foundations have been one of the important subjects in the field of structural and geotechnical engineering to understand the soil-structure interaction phenomenon during earthquake motions.

In the early stage of the study, a single pile in a homogeneous soil layer on rigid base was an object for analysis $^{1) ~ 3)}$. In reality, however, many sites are multi-layered and pile foundations are composed of a number of grouped piles which are connected into a pile cap at their heads ${ }^{4)-5)}$. The grouping effect, which results from the pile-soil-pile interaction, is expected to be rather significant ${ }^{(6)}{ }^{9)}$ so that the single pile analysis cannot always make us lead an appropriate engineering judgement.

In this paper, to investigate a grouped pile foundation in layered soils, the distributed parameter system (DSP) approach is adopted by elaborating the preceding beam analysis in a visco-elastic medium as represented by Refs. 1) - 3). The substructure concept is applied to formulate the 3-dimensional (3-D) soil-grouped pile system; first evaluating the soil dynamic flexibility by imposing a unit time-harmonic force successively at pile cavities and then invert it to find the corresponding soil stiffness which is incorporated into the pile-soil analysis. The coupled motion of soils and piles due to base input, like earthquake input, is solved through the eigenvalue decomposition of complex-valued soil stiffness and the transfer matrix analysis is used to advantage for the pile analysis to account for the variation of soil reaction with depth. The results are presented in terms of the pile head impedance function and the associated driving force (or the so-called effective input). Since the rigorous $3-\mathrm{D}$ analysis consumes much

* Member of JSCE, Dr. Eng., Professor, Department of Civil Engineering, Okayama University, Okayama, 700

** Member of JSCE, M. S., Nihon Dorro Kodan (Japan Highway Public Corporation), Formerly Graduate student 
computing time as the numbers of piles incease, an effective simplified method is also developed based on the "ring-pile" idea. This approach presumes a ring-arrangement of piles in which pile axes on the same radius follow almost a rigid ring movement at any depth. The finite element solution based on the same idea $^{10)}$ is compared with the present DPS solution.

\section{FOURIER SERIES SOLUTION FOR SOIL DYNAMICS}

Soils are modeled by a layered visco-elastic medium. The dynamic equilibrium in a steady state harmonic motion of frequency $\omega$ is governed by the Navior equation,

$\left(\lambda_{j}^{*}+2 \mu_{j}^{*}\right) \nabla\left(\nabla U_{j}\right)-\mu_{j}^{*} \nabla \times \nabla \times U_{j}+\rho_{j} \omega^{2} U_{j}=0$ for the $j$-th layer

in which $\lambda_{j}^{*}$ and $\mu_{j}^{*}$ are complex Lame constants, $\rho_{j}$ is density, $U_{j}$ is the displacement vector and $\nabla$ denotes the gradient operator. When the cylindrical coordinates representation is used, the corresponding solution may be obtained in terms of the Fourier series expansion in the circumferential $\theta$-direction.

$$
\boldsymbol{U}_{j}(r, \theta, z)=\sum_{n=0}^{\infty} \boldsymbol{H}_{n}^{s}(\theta) \hat{\boldsymbol{U}}_{n j}^{s}+\sum_{n=0}^{\infty} \boldsymbol{H}_{n}^{a}(\theta) \hat{\boldsymbol{U}}_{n j}^{a}
$$

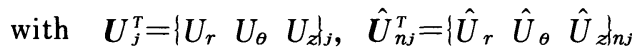

$$
\boldsymbol{H}_{n}^{s}(\theta)=\operatorname{diag}(\cos \widehat{n \theta}-\sin \widehat{n \theta} \cos \widehat{n \theta}), \boldsymbol{H}_{n}^{a}(\theta)=\operatorname{diag}(\sin \widehat{n \theta} \cos \widehat{n \theta} \sin \widehat{n \theta})
$$

in which $\boldsymbol{H}_{n}^{s}$, and $\boldsymbol{H}_{n}^{a}$ denote the symmetric and antisymmetric variation with respect to the $\theta=0^{\circ}(x$-axis), respectively. $\hat{\boldsymbol{U}}_{n}^{s}$ and $\hat{\boldsymbol{U}}_{n}^{a}$ are the corresponding Fourier amplitudes. The time function is omitted.

Herein, in order to simplify the analysis, the authors separated the horizontal and the vertical motions as they are independent each other. In case of no dractic change of deformation in the $\theta$-direction, the solution of Eq. (1) is properly truncated by adopting $n=0$ and 1 terms only. Further, neglecting the effect of torsional motion, Eq. (2) results in

$\boldsymbol{U}_{j}(r, \theta, z)=\boldsymbol{H}_{0}^{s}(\theta) \hat{\boldsymbol{U}}_{0 j}^{s}+\boldsymbol{H}_{1}^{s}(\theta) \hat{\boldsymbol{U}}_{1 j}^{s}+\boldsymbol{H}_{1}^{a}(\theta) \hat{\boldsymbol{U}}_{1 j}^{a}$

in which the first term concerns a vertical motion in $z$-axis direction, the second term concerns a coupled motion of sway along $x$-axis $\left(\theta=0^{\circ}\right)$ and rocking about $y$-axis $\left(\theta=90^{\circ}\right)$, and the third term represents a coupled motion of sway and rocking in the plane perpendicular to the second motion.

Following the Novak and et al's work $\left.{ }^{2)} \sim 4\right)$, the infinitesmal thin layer assumption (equivalently, the plane strain condition) leads the Fourier amplitudes for the above Fourier terms, droping the superfix, of

$$
\left\{\begin{array}{c}
\hat{U}_{r_{1}}(r, z) \\
\hat{U}_{\theta 1}(r, z) \\
\hat{U}_{z 0}(r, z)
\end{array}\right\}_{j}=\left[\begin{array}{ccc}
\frac{\partial}{\partial r} K_{1}(q r) & \frac{1}{r} K_{1}(s r) & 0 \\
\frac{1}{r} K_{1}(q r) & \frac{\partial}{\partial r} K_{1}(s r) & 0 \\
0 & 0 & K_{0}(p r)
\end{array}\right]_{j}\left\{\begin{array}{l}
A_{r}(z) \\
A_{\theta}(z) \\
A_{z}(z)
\end{array}\right\}_{j} \text { or } \hat{\boldsymbol{U}}_{j}(r, z)=\boldsymbol{K}_{j}(r) \boldsymbol{A}_{j}(z) \cdots
$$

in which $K_{m}(\cdot)$ denotes the modified Bessel function of the 2 nd kind of order $m$ that satisfies the boundary condition at infinity $(r \rightarrow \infty)$. The constatnts vector $\boldsymbol{A}_{\boldsymbol{j}}$ is determined from the boundary condition. The parameters involved are defined as

$$
p_{j}=\frac{i\left(\frac{\omega}{V_{s j}}\right)}{\sqrt{1+D_{s j}}}, \quad q_{j}=\frac{i\left(\frac{\omega}{V_{s j}}\right)}{\sqrt{\eta_{j}^{2}+i\left[\left(\eta_{j}^{2}-2\right) D_{V j}+2 D_{s j}\right]}}, \quad s_{j}=\frac{i\left(\frac{\omega}{V_{s j}}\right)}{\sqrt{1+D_{s j}}}
$$

in which $\eta_{j}=V_{p j} / V_{s j}, \quad V_{s j}$ being the shear wave velocity, $V_{p j}$ being the pressure wave velocity and $D_{p j}=D_{s j}=D_{j}$ is the internal damping ratio to be included.

The stress distribution being consistent with the expression of Eq. (3) is given by

$$
\boldsymbol{\sigma}_{j}(r, \theta, z)=\boldsymbol{H}_{0}^{s}(\theta) \hat{\boldsymbol{\sigma}}_{0 j}^{s}+\boldsymbol{H}_{1}^{s}(\theta) \hat{\boldsymbol{\sigma}}_{1 j}^{s}+\boldsymbol{H}_{1}^{a}(\theta) \hat{\boldsymbol{\sigma}}_{1 j}^{a}
$$

The Fourier amplitudes for stresses are found accordingly by differentiating Eq. (4), as

$$
\left\{\begin{array}{c}
\hat{\sigma}_{r 1}(r, z) \\
\hat{\tau}_{r \theta 1}(r, z) \\
\hat{\tau}_{r z 0}(r, z)
\end{array}\right\}_{j}=
$$




$$
\left.\left[\begin{array}{ccc}
\left(\lambda^{*}+2 \mu^{*}\right) q^{2} K_{1}(q r)+\frac{2 \mu^{*}}{r} K_{0}(q r)+\frac{4 \mu^{*}}{r^{2}} K_{1}(q r) & -2 \mu^{*}\left\{\frac{s}{r} K_{0}(s r)+\frac{2}{r^{2}} K_{1}(s r)\right\} & 0 \\
-2 \mu^{*}\left\{\frac{q}{r} K_{0}(q r)+\frac{2}{r^{2}} K_{1}(q r)\right\} & \mu^{*}\left(s^{2}+\frac{4}{r^{2}}\right) K_{1}(s r)+\frac{2 \mu^{*}}{r} s K_{0}(s r) & 0 \\
0 & 0 & \left.\mu^{*} \frac{\partial K_{0}(p r)}{\partial r}\right]_{j}
\end{array}\right\}_{j}^{A_{z}(z)}\right)_{j}
$$

or

$$
\hat{\sigma}_{j}(r, z)=J_{j}(r) A_{j}(z)
$$

in which the superfix $s, a$ are again omitted. Eliminating the unknown constants vector $\boldsymbol{A}_{j}$ from Eqs. (4) and $(6)$, one can get

$$
\hat{\boldsymbol{U}}_{j}(r, z)=\boldsymbol{K}_{j}(r) \boldsymbol{J}_{j}^{-1}(r) \hat{\sigma}_{j}(r, z)
$$

Note here that this relation is applicable to calcurate the displcements at radius $r_{1}$ due to the stress distribution $\hat{\sigma}_{j}$ at the radius $r_{2}\left(<r_{1}\right)$. Hence,

$$
\hat{\boldsymbol{U}}_{j}\left(r_{1}, z\right)=\boldsymbol{K}_{j}\left(r_{1}\right) \boldsymbol{J}_{j}^{-1}\left(r_{2}\right) \hat{\sigma}_{j}\left(r_{2}, z\right)
$$

\section{SOIL FLEXIBILITY}

Consider the soil layers with pile cavities after piles are taken off but with stress distribution along the pile cavities which is in equilibrium with pile internal forces. Prior to finding the soil stiffness associated with the pile nodes, one may establish the corresponding soil flexibility first. This is calculated by imposing a unit time-harmonic force at the respective pile nodes in a specified direction successively. When rigid sections are assumed for pile cavities, the solution of Eq. ( 8 ) can be utilized on the plane strain condition. The stress distribution along pile cavities which equilibriates with unit forces acting in $x, y$ and $z$ directions at I-point (making a tentative origin at this point in the Cartesian coordinates) are found from

$$
\begin{aligned}
& P_{x}=\int_{0}^{2 \pi}\left(\sigma_{r}\left(r_{0}\right) \cos \theta-\tau_{r \theta}\left(r_{0}\right) \sin \theta\right) r_{0} d \theta=1 \\
& P_{y}=\int_{0}^{2 \pi}\left(\sigma_{r}\left(r_{0}\right) \sin \theta+\tau_{r \theta}\left(r_{0}\right) \cos \theta\right) r_{0} d \theta=1 \\
& P_{z}=\int_{0}^{2 \pi} \tau_{r z}\left(r_{0}\right) r_{0} d \theta=1 \ldots \ldots \ldots \ldots \ldots \ldots \ldots \ldots \ldots \ldots \ldots \ldots \ldots \ldots \ldots
\end{aligned}
$$

in which $r_{0}$ is the pile radius. From Eq. ( 8 ), one can calculate the response at certain poin $\mathrm{J}$ with radius $r\left(>r_{0}\right)$ and angle $\theta$ counter-clockwise from $x$-axis due to a unit time-harmonic force at the I-pont acting in the $x$-direction.

$$
\left\{\begin{array}{l}
U_{x x}^{J I} \\
U_{y x}^{J I}
\end{array}\right\}_{j}=\left[\begin{array}{cc}
c^{2} & s^{2} \\
c s & -c s
\end{array}\right]\left\{\begin{array}{l}
\hat{U}_{r 1}^{s} \\
\hat{U}_{\theta 1}^{s}
\end{array}\right\}_{j} \text { with } c=\cos \theta, s=\sin \theta
$$

in which the symmetric Fourier harmonic of $n=1$ is taken (Fig. 1(a)). The response due to a unit time-harmonic force acting in the $y$-direction is likewise given by

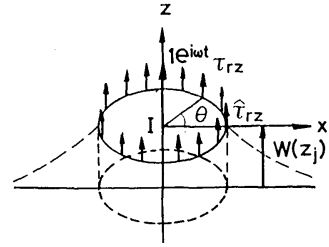

Z-Direction

(a) $X$-Direction

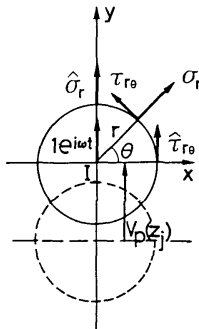

Y-Direction

(b) $Y$-Direction

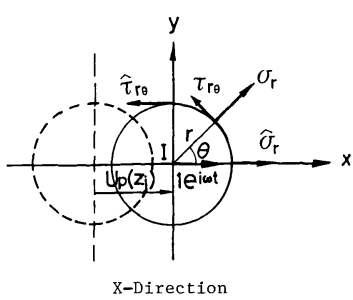

(c) Z-Direction

Fig. 1 Stress Distributions along Pile Cavities 


$$
\left\{\begin{array}{l}
U_{x y}^{J} \\
U_{y y}^{J I}
\end{array}\right\}_{j}=\left[\begin{array}{cc}
c s & -c s \\
s^{2} & c^{2}
\end{array}\right]\left\{\begin{array}{c}
\hat{U}_{r 1}^{a} \\
\hat{U}_{\theta 1}^{a}
\end{array}\right\}_{j}
$$

in which the antisymmetric Fourier harmonic of $n=1$ is chosen (Fig. 1(b)). The response in the $z$-direction due to a unit time-harmonic force in the same direction results, by using the symmetric Fourier harmonic of $n=0$ (Fig. 1( c)).

$$
U_{z j}^{J I}=\hat{U}_{z 0 j}^{s}
$$

The dynamic flexibility elements between I and $J$ points are then assembled from Eqs. (12) through (14). Hence, the total flexibility matrix regarding interface nodes with piles results.

$$
\boldsymbol{F}_{x y z, j}^{\mathrm{soi1}}=\left[\begin{array}{cccc}
\boldsymbol{F}_{x y z}^{11} & \boldsymbol{F}_{x y z}^{12} & \cdots & \boldsymbol{F}_{x y z}^{1 N \rho} \\
& \boldsymbol{F}_{x y z}^{22} & & \vdots \\
& \text { Sym } & \cdots & \boldsymbol{F}_{x y z}^{N o N p}
\end{array}\right]_{j}
$$

in which

$$
\boldsymbol{F}_{x y z, j}^{J I}=\left[\begin{array}{ccc}
U_{x x}^{J I} & U_{y x}^{J I} & \\
U_{x y}^{J I} & U_{y y}^{J I} & \\
& & U_{z}^{J I}
\end{array}\right],
$$

and $N_{\rho}$ is the numbers of piles. As the inverse of the flexibility matrix, one can define the dynamic soil stiffness.

$$
\boldsymbol{K}_{x y z, j}^{\mathrm{soi1}}=\left[\begin{array}{ll}
\boldsymbol{K}_{x y}^{\mathrm{soil}} & \\
& \boldsymbol{K}_{z}^{\mathrm{soil}}
\end{array}\right]_{j}=\left(\boldsymbol{F}_{x y z, j}^{\mathrm{soi1}}\right)^{-1}
$$

in which the total dynamic stiffness is partitioned into the independent ones as indicated by the suffix.

\section{3-DIMENSIONAL PILE-SOIL-PILE INTERACTION}

Lateral Vibration : The coupled motion of grouped piles with the neighboring soil, when the base input $U_{g}$ is imposed at their tips, are governed by

$$
\boldsymbol{M}_{p} \frac{\partial^{2} \boldsymbol{U}_{p j}}{\partial t^{2}}+\boldsymbol{E}_{p} \boldsymbol{I}_{p} \frac{\partial^{4} \boldsymbol{U}_{p, j}}{\partial z^{4}}+\boldsymbol{K}_{x y, j}^{\text {soil }} \boldsymbol{U}_{p, j}=\boldsymbol{K}_{x y, j}^{\text {soil }} \boldsymbol{U}_{s, j} \text { for the segment in } j \text {-th soil layer }
$$

in which $\boldsymbol{M}_{p}$ is the diagonal mass matrix of order $2 N_{p}, \boldsymbol{E}_{p} \boldsymbol{I}_{p}$ is the diagonal bending stiffness matrix of piles, $\boldsymbol{U}_{p}$ is the pile displacements having components in $x$ and $y$ directions, $\boldsymbol{U}_{s j}$ is the soil displacements as a free field. The soil reaction is incorporated from the soil stiffness of Eq. (17). Note that the piles are coupled through the soil stiffness $\boldsymbol{K}_{x y, j}^{\text {soil. }}$ The general solution of Eq. (18) is given by superposing the homogeneous one when there is no free field displacement and the particular solution due to it.

$$
\boldsymbol{U}_{p, j}(z)=\boldsymbol{U}_{p, j}^{0}(z)+\boldsymbol{U}_{p, j}^{*}(z)
$$

The homogeneous equation is now decoupled into independent governing equations through the eignevalue procedure of

$$
\left(\beta_{j}-\alpha^{4} \boldsymbol{I}\right) \boldsymbol{U}_{p, j}^{0}=0 \quad \text { with } \quad \beta_{j}=\left(\boldsymbol{E}_{p} \boldsymbol{I}_{p}\right)^{-1}\left(\omega^{2} \boldsymbol{M}_{p}-\boldsymbol{K}_{x y, j}^{\mathrm{soi}}\right)
$$

which yields $2 N_{p}$ eigenvalues of $\alpha_{l}^{m}\left(=\alpha_{l},-\alpha_{l}, i \alpha_{l},-i \alpha_{l}\right)$. The pile displacements are then expressed as

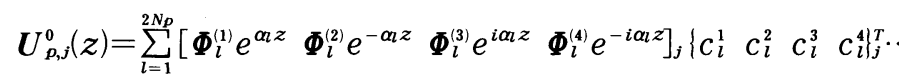

in which $\Phi_{l}^{(m)}$ is the eigenvector associated with $\alpha_{l}^{m}$, and $c_{l}^{m}$ are the participation coefficients. The particular solution is found from

$$
\boldsymbol{U}_{p, j}^{*}(\boldsymbol{z})=\left(\boldsymbol{k}_{j}^{4} \boldsymbol{E}_{p} \boldsymbol{I}_{p}-\omega^{2} \boldsymbol{M}_{p}+\boldsymbol{K}_{x y, j}^{\mathrm{soil}}\right)^{-1} \boldsymbol{K}_{x y, j}^{\mathrm{soil}} \boldsymbol{U}_{s} \text { with } \boldsymbol{k}_{j}=\frac{i \omega}{V_{s j}}
$$

in which the free field displacement is solved from the 1-dimensional shear wave equation by use of the transfer matrix method.

The transfer matrix method is also applied for the decoupled piles analysis ${ }^{5)}$. The piles are devided into a finite number of segments as to comply with the surrounding soil layers of different properties. From the 
elementary beam theory, the slopes $\boldsymbol{\theta}_{p}$, bending moments $M_{p}$, shearing forces $\boldsymbol{Q}_{p}$ are obtained by differentiating Eq. (19), which yields a matrix of derivatives $\boldsymbol{S}(z)$. The quantities at the upper section of $j$-th segment are expressed through the matrix $S$ in terms of those at the lower section of the same segments.

$$
\tilde{\boldsymbol{U}}_{p, j}^{0}(0)=\tilde{\boldsymbol{T}}_{j} \cdot\left(\tilde{\boldsymbol{U}}_{p j}^{0}\left(H_{j}\right)-\tilde{\boldsymbol{U}}_{p, j}^{*}\left(H_{j}\right)\right)+\tilde{\boldsymbol{U}}_{p, j}^{*}(0)
$$

in which

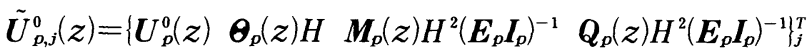

$$
\begin{aligned}
& \tilde{U}_{p, j}^{*}(z)=\left\{\begin{array}{llll}
\boldsymbol{U}_{p}^{*}(z) & \boldsymbol{U}_{p}^{* \prime}(z) H & \boldsymbol{U}_{p}^{* \prime \prime}(z) H^{2}\left(\boldsymbol{E}_{p} \boldsymbol{I}_{p}\right)^{-1} & \left.\boldsymbol{U}_{p}^{* \prime \prime \prime}(z) H^{3}\left(\boldsymbol{E}_{\rho} \boldsymbol{I}_{p}\right)^{-1}\right\}_{j}^{T}
\end{array}\right. \\
& \tilde{\boldsymbol{T}}_{j}=\operatorname{diag}\left(1 / \xi_{j} 1 / \xi_{j}^{2} 1 / \xi_{j}^{3}\right) \cdot \boldsymbol{S}_{j}(0) \boldsymbol{S}_{j}^{-1}\left(H_{j}\right) \cdot \operatorname{diag}\left(1 \quad \xi_{j} \xi_{j}^{2} \quad \xi_{j}^{3}\right)
\end{aligned}
$$

and $\xi_{j}=H_{j} / H ; H=\sum_{j=1}^{N_{s}} H_{j}$, and $H_{j}$ 's are the $j$-th layer thickness, $N_{s}$ is the numbers of soil layers and $H$ is the total soil depth. Applying the above relationship to all the segments, one can relate the pile head quantities with the pile tip ones.

$$
\boldsymbol{U}_{p, 1}(0)=\prod_{j=1}^{N_{s}} \tilde{\boldsymbol{T}}_{j} \tilde{\boldsymbol{U}}_{p, N_{s}}\left(H_{N_{S}}\right)+\sum_{j=1}^{N_{s}} \prod_{j=1}^{j-1} \tilde{\boldsymbol{T}}_{j}\left(-\tilde{\boldsymbol{T}}_{j} \tilde{\boldsymbol{U}}_{p, j}^{*}\left(H_{j}\right)+\tilde{\boldsymbol{U}}_{\rho, j}^{*}(0)\right)
$$

Substituting the pile tip condition into the above equation and rearranging the result, one can develop the relationship between the internal forces and deformations at the pile head only.

$$
\left\{\begin{array}{c}
\boldsymbol{M}_{p}(0) H^{2}\left(\boldsymbol{E}_{\rho} \boldsymbol{I}_{p}\right)^{-1} \\
\boldsymbol{Q}_{p}(0) H^{3}\left(\boldsymbol{E}_{\rho} \boldsymbol{I}_{\rho}\right)^{-1}
\end{array}\right\}_{1}=\left[\begin{array}{ll}
\boldsymbol{K}_{\theta x}^{h} & \boldsymbol{K}_{\theta \theta}^{h} \\
\boldsymbol{K}_{x x}^{h} & \boldsymbol{K}_{x \theta}^{h}
\end{array}\right]\left(\left\{\begin{array}{c}
\boldsymbol{U}_{\rho}(0) \\
\boldsymbol{\theta}(0) H
\end{array}\right\}_{1}-\left\{\begin{array}{c}
\boldsymbol{D}_{1} \\
\boldsymbol{D}_{2}
\end{array}\right\} U_{g}\right)+\left\{\begin{array}{c}
\boldsymbol{D}_{3} \\
\boldsymbol{D}_{\mathbf{4}}
\end{array}\right\} U_{\boldsymbol{g}}
$$

in which the matric $K_{i j}^{h}$, depending on the pile tip condition, defines the pile head impdedance functions and, the vector $\boldsymbol{D}=\left\{\begin{array}{lllll}\boldsymbol{D}_{1} & \boldsymbol{D}_{2} & \boldsymbol{D}_{3} & \boldsymbol{D}_{4}\end{array}\right\}$ represents the summation term in Eq. (24), and the expression

$$
\boldsymbol{P}_{p}^{h 0}=\left(-\left[\begin{array}{ll}
\boldsymbol{K}_{\theta x}^{h} & \boldsymbol{K}_{\theta \theta}^{h} \\
\boldsymbol{K}_{x x}^{h} & \boldsymbol{K}_{x \theta}^{h}
\end{array}\right]\left\{\begin{array}{c}
\boldsymbol{D}_{1} \\
\boldsymbol{D}_{2}
\end{array}\right\}+\left\{\begin{array}{c}
\boldsymbol{D}_{3} \\
\boldsymbol{D}_{4}
\end{array}\right\}\right) U_{g}
$$

gives the associated driving force at pile head (the effective input) due to the base motion $U_{g}$.

Longitudinal Vibration : The governing equations for grouped piles are also coupled through the soil stiffness $\boldsymbol{K}_{z, j}^{\text {soil }}$ as

$$
\boldsymbol{M}_{p} \frac{\partial^{2} \boldsymbol{W}_{p, j}}{\partial z^{2}}-\boldsymbol{E}_{p} \boldsymbol{A}_{p} \frac{\partial^{2} \boldsymbol{W}_{p, j}}{\partial z^{2}}+\boldsymbol{K}_{z, j}^{\mathrm{soil}} \boldsymbol{W}_{p, j}=\mathbf{0}
$$

in which $\boldsymbol{E}_{p} \boldsymbol{A}_{p}$ is the diagonal axial stiffness matrix of piles, $\boldsymbol{W}_{p}$ is the vector of axial deformation. Again, the decoupling is executed through the eigenvalue problem of

$$
\left(\gamma_{j}+\alpha^{2} \boldsymbol{I}\right) \boldsymbol{W}_{\rho, j}=\mathbf{0} \quad \text { with } \quad \gamma_{j}=\left(\boldsymbol{E}_{\rho} \boldsymbol{A}_{p}\right)^{-1}\left(\boldsymbol{K}_{z, j}^{\text {soil }}-\omega^{2} \boldsymbol{M}_{\rho}\right)
$$

The solution is then

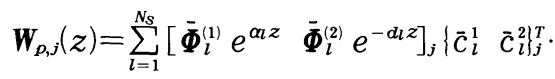

Associated with the deformation $\boldsymbol{W}_{\rho, j}$ and axial force $\boldsymbol{N}_{\rho, j}$, one can define a matrix of derivatives $\bar{S}_{j}(z)$.

The transfer matrix of the piles for the $j$-th segment becomes as

$$
\tilde{\boldsymbol{W}}_{p, j}(0)=\overline{\boldsymbol{T}}_{j} \cdot \tilde{\boldsymbol{W}}_{p, j}\left(H_{j}\right)
$$

in which

$$
\tilde{\boldsymbol{W}}_{\rho, j}(z)=\left\{\boldsymbol{W}_{\rho}(z) N_{\rho}(z)\left(E_{\rho} A_{\rho}\right)^{-1}\right\}_{j}^{T}
$$

This application from the pile head to tip results in

$$
\tilde{\boldsymbol{W}}_{p, 1}(0)=\prod_{j=1}^{N_{S}} \overline{\boldsymbol{T}}_{j} \cdot \tilde{\boldsymbol{W}}_{p, N_{S}}\left(H_{N_{S}}\right)
$$

Substituting the condition at pile tip being constraint in the vertical direction yields

$$
\boldsymbol{N}_{\rho, 1}(0) H\left(\boldsymbol{E}_{p} \boldsymbol{A}_{p}\right)^{-1}=\boldsymbol{K}_{z}^{h} \boldsymbol{W}_{\rho, 1}(0)
$$

in which $\boldsymbol{K}_{z}^{h}$ defines the pile head impedance. 
Grouped Pile Impedance and Effective Input : Consider the pile cap motion in $x z$-plane. This structural part is assumed to be a rigid body ; therefore, the respective pile head characteristics are forced to obey the degrees freedom of this part. Here in the coupled motion of sway and rocking are accounted for at its gravity center (G. C. ) so that the pile head deformation $U_{p}^{h}$ is transfterred to the cap motion $U_{F}$ through the geometric master-slave relationship. Referring to Fig. 2, this is given by

$$
\tilde{\boldsymbol{T}}_{p}=\left[\begin{array}{lll}
\boldsymbol{T}_{p 1} & \boldsymbol{T}_{p 2} \cdots \boldsymbol{T}_{p N_{p}}
\end{array}\right] \text { with } \quad \boldsymbol{T}_{p l}=\left[\begin{array}{ccc}
1 & 0 & 0 \\
-z_{G} & 1 & x_{p}
\end{array}\right] \cdots
$$

Assembling the pile head impedances $K_{p}^{h}$ from Eqs. (25) and (33), one can express the pile head force-deformation relationship. The gross effect is then summed up from individual pile contributions, leading the total pile head impedance and the corresponding driving force (effective input) respectively, as

$$
\begin{aligned}
& \boldsymbol{K}_{F}=\tilde{\boldsymbol{T}}_{p}^{T} \boldsymbol{K}_{p}^{h} \tilde{\boldsymbol{T}}_{p} \\
& \boldsymbol{P}_{p}^{0}=\tilde{\boldsymbol{T}}_{p}^{T} \boldsymbol{P}_{p}^{h 0} \ldots
\end{aligned}
$$

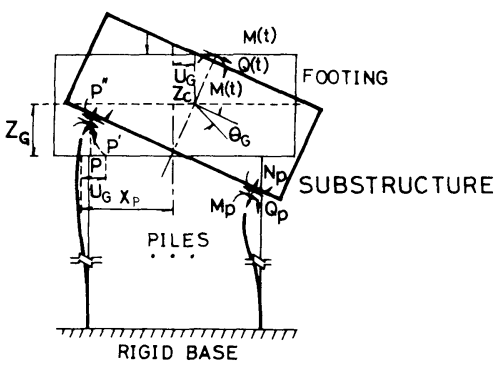

Fig. 2 Grouped Piles-Rigid Footing System

\section{SIMPLIFIED RING-PILE ANALYSIS}

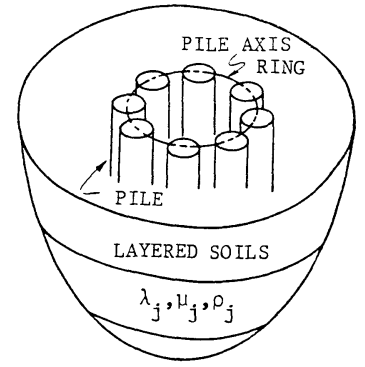

Fig. 3 Ring-Piles

In the case of piles being in a concentric arrangement (see Fig. 3), the following simplified analysis may be effective. For the motion of sway and rocking of global soil-piles movement in the $x z$-plane, one may approximate it by a symmetric Fourier harmonic of $n=1$. The soil displacements at radius $r_{J}$ connecting concentric pile cavities due to the stresses at radius $r_{I}\left(<r_{J}\right)$ connecting another concentric pile cavities, are similarly derived as Eq. ( 8 ) but are replaced by

$$
\hat{\boldsymbol{U}}_{j}\left(r_{J}, z\right)=\overline{\boldsymbol{K}}_{j}\left(r_{J}\right) \overline{\boldsymbol{J}}_{j}^{-1}\left(r_{I}\right) \hat{\boldsymbol{\sigma}}\left(r_{l} \cdot z\right)
$$

in which the elements of $\boldsymbol{K}_{j}(r)$ and $\boldsymbol{J}_{j}(r)$ in Eqs. (4) and (6) are modified accordingly such that

$$
\begin{aligned}
& \overline{\boldsymbol{K}}_{j}(r)=\left[\begin{array}{l:l}
\text { Identical with } \\
\text { elements in } \boldsymbol{K}(r) \text { in Eq. } 4 & \\
\hdashline & K_{1}(p r)
\end{array}\right]_{j} \\
& \bar{J}_{j}(r)=\left[\begin{array}{l:c}
\text { Identical with } & \\
\text { elements in } \boldsymbol{J}(r) \text { in Eq. } 6 & \\
\hdashline & \mu^{*} \frac{\partial K_{1}(p r)}{\partial r}
\end{array}\right]_{j}
\end{aligned}
$$

When the forces per unit radian $\hat{\boldsymbol{P}}_{j}$ at $r_{I}$ is used instead of stress distirbutions, Eq. (37) becomes

$$
\hat{\boldsymbol{U}}_{j}\left(r_{J}, z\right)=\left(1 / r_{\mathrm{I}}\right) \overline{\boldsymbol{K}}_{j}\left(r_{J}\right) \overline{\boldsymbol{J}}_{j}^{-1}\left(r_{I}\right) \hat{\boldsymbol{P}}_{j}\left(r_{I}, z\right)
$$

Making use of this relation for every concentric pile cavities, one can get the soil flexibility defined at ring-pile axes.

$$
\overline{\boldsymbol{F}}_{r \boldsymbol{z}, j}^{\mathrm{soi1}}=\left[\begin{array}{cccc}
\frac{1}{r_{1}} \overline{\boldsymbol{K}}\left(r_{1}\right) \overline{\boldsymbol{J}}^{-1}\left(r_{1}\right) & & \\
\frac{1}{r_{1}} \overline{\boldsymbol{K}}\left(r_{2}\right) \overline{\boldsymbol{J}}^{-1}\left(r_{1}\right) & \frac{1}{r_{2}} \overline{\boldsymbol{K}}\left(r_{2}\right) \overline{\boldsymbol{J}}^{-1}\left(r_{2}\right) & \text { sym } \\
\vdots & & \ddots & \\
\frac{1}{r_{1}} \overline{\boldsymbol{K}}\left(r_{N_{s}}\right) \overline{\boldsymbol{J}}^{-1}\left(r_{1}\right) & \ldots \ldots \ldots & \frac{1}{r_{N_{p}}} \overline{\boldsymbol{K}}\left(r_{N_{p}}\right) \overline{\boldsymbol{J}}^{-1}\left(r_{N_{p}}\right)
\end{array}\right]_{j}
$$


Note here that the modified Bessel function involved are only valid for the elements in the lower triangle matrix. However, from the knowledge of the Maxwell's reciprocal theorem, $\overline{\boldsymbol{F}}_{r \theta z, j}^{\mathrm{soil}}$ has a symmetric nature, which is used to advantage to make Eq. (41) full. The corresponding soil stiffness $\overline{\boldsymbol{K}}_{r \theta z, j}^{\text {soil }}$ is computed from the inverse so that

$$
\overline{\boldsymbol{K}}_{r \theta z, j}^{\mathrm{soil}}=\left[\begin{array}{ll}
\overline{\boldsymbol{K}}_{r \theta}^{\text {soil }} & \\
& \overline{\boldsymbol{K}}_{z}^{\text {soil }}
\end{array}\right]_{j}=\left(\overline{\boldsymbol{F}}_{r \theta z, j}^{\mathrm{soil}}\right)^{-1}
$$

in which the partitioning is carried out from the independency of elements.

The governing equation of the lateral deformation of ring-piles $\left(N_{r}\right.$ piles $)$ at radius $r$ is now obtained, as a gross along the circumferential direction, by claiming the compatibility and equilibrium in the sense of integral over the ring, which is called a "ring-pile" analysis herein,

$$
\begin{aligned}
& \sum_{l=1}^{N r}\left[\begin{array}{cc}
c_{l}^{2} & s_{l}^{2} \\
s_{l} c_{l} & -s_{l} c_{l}
\end{array}\right]\left(-\omega^{2} M_{\rho}\left\{\begin{array}{c}
\hat{\boldsymbol{U}}_{r} \\
\hat{\boldsymbol{U}}_{\theta}
\end{array}\right\}_{r}+\boldsymbol{E}_{\rho} \boldsymbol{I}_{p} \frac{\partial^{4}}{\partial z^{4}}\left\{\begin{array}{c}
\hat{\boldsymbol{U}}_{r} \\
\hat{\boldsymbol{U}}_{\theta}
\end{array}\right\}_{r}\right)_{j}+\int_{0}^{2 \pi}\left[\begin{array}{cc}
c^{2} & s^{2} \\
s c & -s c
\end{array}\right]\left\{\begin{array}{l}
\hat{\boldsymbol{P}}_{r} \\
\hat{\boldsymbol{P}}_{\theta}
\end{array}\right\}_{r_{j}} d \theta \\
& =\int_{0}^{2 \pi}\left[\begin{array}{cc}
c^{2} & s^{2} \\
s c & -s c
\end{array}\right]\left\{\begin{array}{l}
\hat{\boldsymbol{P}}_{r}^{\text {soil }} \\
\hat{\boldsymbol{P}}_{\theta}^{\text {soil }}
\end{array}\right\}_{r j} d \theta
\end{aligned}
$$

In view of the axisymmetric nature of soil-pile system, and introducing the soil reaction from $\mathrm{Eq}$. (42), one can get for all the ring-piles as

$$
\left[\left(-\omega^{2} \frac{N_{r}}{2} M_{\rho}\right)_{r}\right]_{j}\left\{\left\{\begin{array}{c}
\hat{\boldsymbol{U}}_{r} \\
\hat{\boldsymbol{U}}_{\theta}
\end{array}\right\}_{r}\right\}_{j}+\left[\left(\frac{N_{r}}{2} \boldsymbol{E}_{\rho} \boldsymbol{I}_{p}\right)_{r}\right]_{j}\left\{\frac{\partial^{4}}{\partial z^{4}}\left\{\begin{array}{c}
\hat{\boldsymbol{U}}_{r} \\
\hat{\boldsymbol{U}}_{\theta}
\end{array}\right\}_{r}\right\}_{j}+\pi \overline{\boldsymbol{K}}_{r \theta, j}^{\text {soil }}\left\{\left\{\begin{array}{c}
\hat{\boldsymbol{U}}_{r} \\
\hat{\boldsymbol{U}}_{\theta}
\end{array}\right\}_{r}\right\}_{j}=\pi \overline{\boldsymbol{K}}_{r \theta, j}^{\text {soil }}\left\{\left\{\begin{array}{c}
1 \\
1
\end{array}\right\}_{r} \boldsymbol{U}_{s}\right\}_{j}
$$

in which the coefficients $\pi$ and $N_{r} / 2$ are the results of mathematical operation of the trigonometric functions involved, and the 1-D free field response $\boldsymbol{U}_{s}$ is transferred into the Fourier symmetric term of $n$ $=1$.

The eigenvalue technique is used to decouple Eq. (44). The subsequent procedure is the same for the 3 -D analysis with use of the transfer matrix method.

The ring-pile longitudinal vibration is also accounted for approximately by taking a symmetric Fourier term of $n=1$ so that for the ring-pile on radius $r$

$$
\sum_{l=1}^{N r} c_{l}^{2}\left(-\omega^{2} \boldsymbol{M}_{p} \hat{\boldsymbol{U}}_{z, r_{j}}-\boldsymbol{E}_{p} \boldsymbol{A}_{p} \frac{\partial^{2} \hat{\boldsymbol{U}}_{z, r_{j}}}{\partial z^{2}}\right)+\int_{0}^{2 \pi} c^{2} \hat{\boldsymbol{P}}_{z, r_{j}} d \theta=0
$$

After executing the operation with the symmetric nature in mind, one can get for all the ring-piles

$$
\left[\left(-\omega^{2} \frac{N_{r}}{2} M_{p}\right)_{r}\right]_{j}\left\{\hat{\boldsymbol{U}}_{z, \gamma_{j}}+\left[\frac{N_{r}}{2}\left(\boldsymbol{E}_{p} \boldsymbol{A}_{p}\right)_{r}\right]_{j}\left\{\frac{\partial^{2} \boldsymbol{U}_{z, r}}{\partial z^{2}}\right\}_{j}+\pi \overline{\boldsymbol{K}}_{z j}^{\text {soil }\left\{\boldsymbol{U}_{z, \gamma_{j}}=\mathbf{0}\right.}\right.
$$

in which the dynamic soil stiffness in the vertical direction is substituted from Eq. (42). The eigenvalue techinique is then taken.

Once all the pile head impedance $\boldsymbol{K}_{r p}^{h}$ and the associated driving force $\boldsymbol{P}_{r p}^{h 0}$ are obtained, they are transferred to those at the footing G. C. by

$$
\begin{aligned}
& \overline{\boldsymbol{K}}_{F}=\tilde{\boldsymbol{T}}_{r p}^{T} \boldsymbol{K}_{r p}^{h} \tilde{\boldsymbol{T}}_{r p} . \\
& \overline{\boldsymbol{P}}_{F}^{0}=\tilde{\boldsymbol{T}}_{r p}^{T} \boldsymbol{P}_{r p}^{h 0} \ldots \ldots
\end{aligned}
$$

in which the tranfer matrix is given by

$$
\tilde{\boldsymbol{T}}_{r p}=\left[\begin{array}{llll}
\boldsymbol{T}_{r p 1} & \left.\boldsymbol{T}_{r p 2} \cdots \boldsymbol{T}_{r \rho N}\right]
\end{array}\right] \text { with } \boldsymbol{T}_{r p}=\left[\begin{array}{ccccc}
1 & 1 & 0 & 0 & 0 \\
-z_{G} & -z_{G} & 1 & 1 & r_{P}
\end{array}\right]
$$

\section{NUMERICAL RESULTS}

As an illustrative example, a bridge foundation as shown in Fig. 4 is analyzed.

The pile head impedance function, being directly concerned with the inertial interaction with the 
structure, is first investigated. In Figs. 5, the present 3-D solution from Eq. (35) is compared with the approximate ring-pile solution from Eq. (47). It is noted that both solutions give a good agreement in the low frequency range below $5 \mathrm{~Hz}$, which is the most important range from the view point of earthquake response analysis. The pseudo-3-D finite element solution from the companion paper ${ }^{10)}$, which takes the ring-pile assumption, is also depicted in the same figure. The comparison between solutions from the present ring-pile analysis and from the above finite element method gives the difference in evaluating soil reaction. The fact that a good agreement of these solutions is attained substantiates adopting the plane strain assumption for soil reaction. Beyond $5 \mathrm{~Hz}$, the ring-pile solution deviates from the 3-D solution and loses its accuracy. This may be interpreted that the vibration feature becomes very complicated in this high frequency range and the Fourier harmonics expansion of $n=1$ only for the motion of concentric pile axes fails to approximate the original behavior. In Figs. 5 the results from the single pile analysis are also plotted. Noted is the large deviation from the 3-D solution due to the pile grouping effect.

In order to look into the pile grouping effect further, the following indeces are evaluated:

$e_{N_{p}}^{q}=Q_{N_{p}} / N_{p} Q_{0} \quad e_{N_{p}}^{m}=M_{N_{p}} / N_{p} M_{0}$

which define the ratios of the shear force and the bending moment respectively, as a grouped piles to the sum of single piles, to produce a unit horizantal sway and rotation separately at the pile cap G. C. in a coupled state of sway and rocking motion. Note in Fig. 6 that the dynamic grouping effect varies

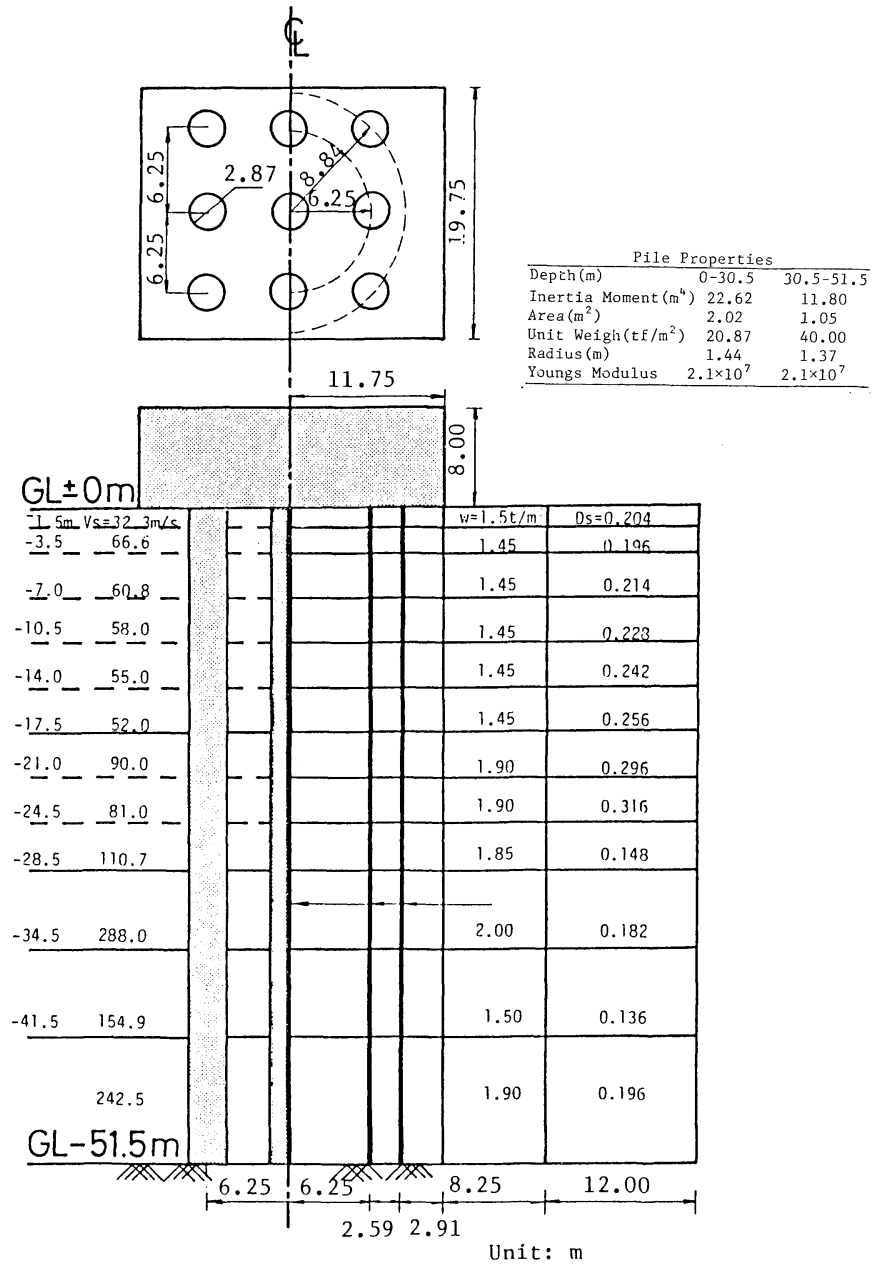

Fig. 4 Grouped Pile Foundation
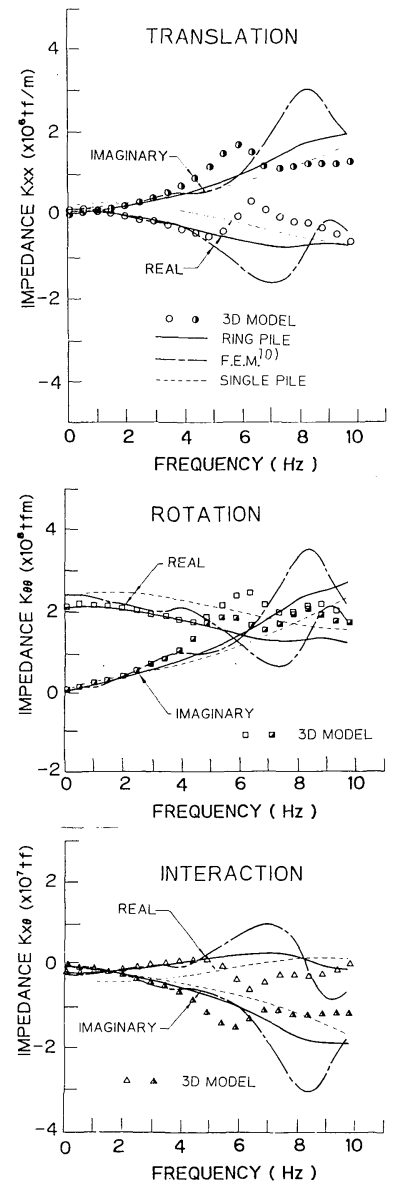

Fig. 5 Grouped Pile Head Impedance Functions 
significantly with the driving frequency. In the very low frequency range below $2 \mathrm{~Hz}$, it remains almost constant value of being less than 1 but it grows in the mid-frequency range between $4-8 \mathrm{~Hz}$, indicating a peak in this range. In view of this tendency, the static result for grouping effect may be of use in the seismic analysis only when the predominant frequency of soil-structure interaction system is very low but it may not be appropriate in other situation.

In designing pile foundation, an interest may be placed on the load distribution from pile cap to individual piles. Figs. 7 show the internal forces induced in the respective pile head when a

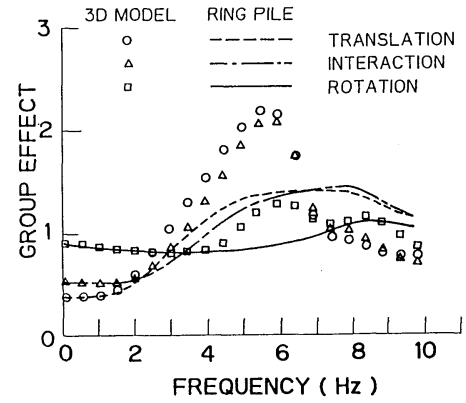

Fig. 6 Group Effect; $\left|e_{N_{p}}^{q}\right|,\left|e_{N_{p}}^{m}\right|$. unit harmonic horizontal force is imposed on the pile cap G. C. The comparison between the 3-D and the ring-pile solutions in distributed parameter system approach indicates that piles in a long distance from the center give a better agreement than those in a short distance. In the low frequency range the greater internal forces are induced in the former piles than in the latter ones. This trend, however, is reversed as the frequency becomes high. Figs. 8 correspond to the load distribution to respective pile head when a unit hamonic moment acts at the pile cap G. C. The 3-D and ring-pile solution coincide well. Note that the moment is resisted by the axial force of piles in a long distance from the center rather than the bending moment at pile heads. The frequency dependency is not so strong as in the horizontal force case.

Figs. 9 show the transmitting function of the driving force from base to pile cap G. C. through the soil-pile interaction. The grouping effect is noted to be appreciable in the very low frequency range. The single pile analysis results in a greater amplification than the grouped pile analysis. This is understood in veiw of the reduced grouped pile head stiffness from the sum of the single pile head stiffness in this
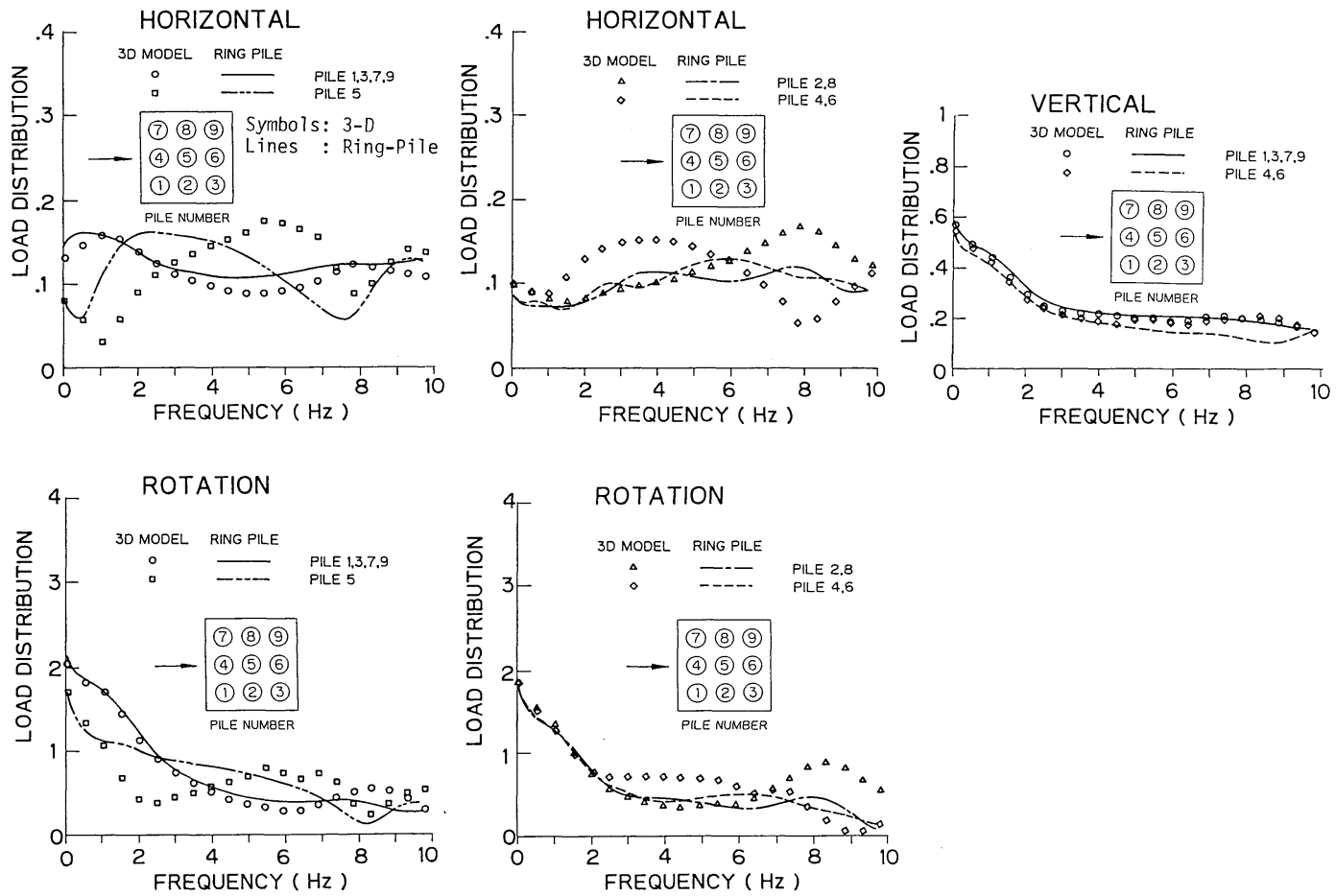

Fig. 7 Pile Head Internal Forces due to Unit Horizontal Harmonic Load at Pile Cap 

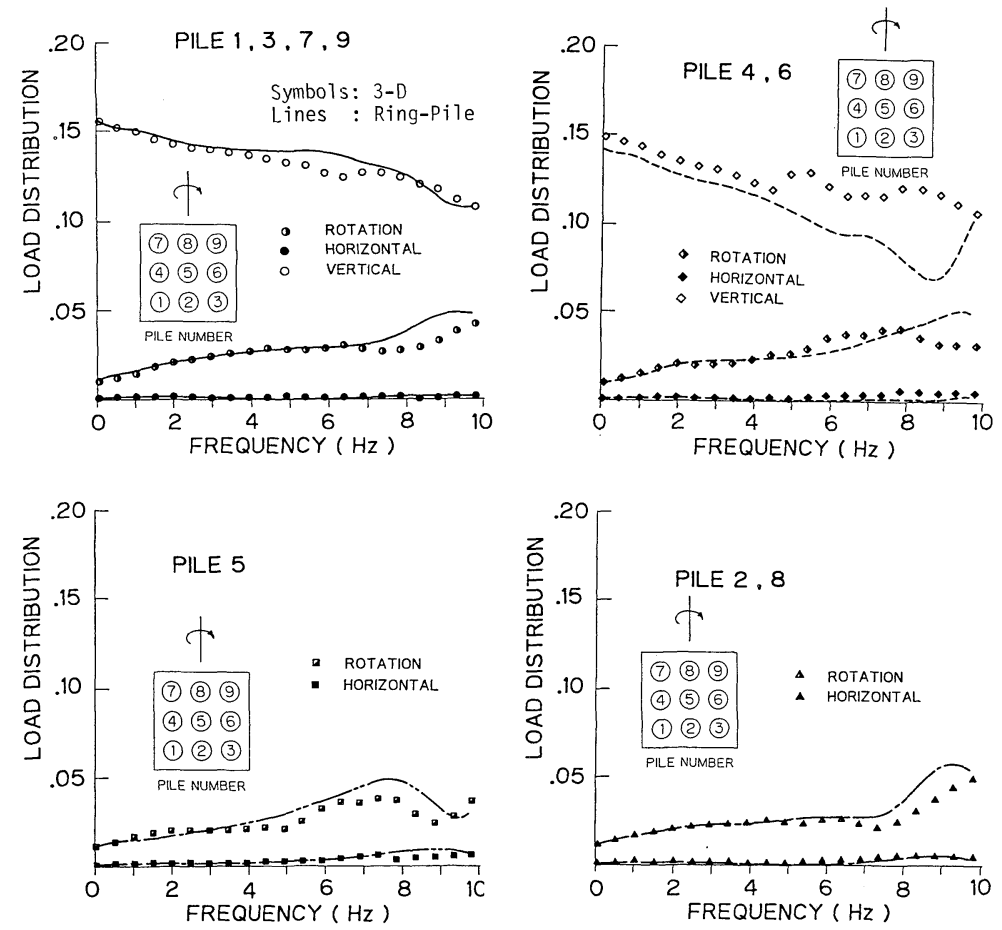

Fig. 8 Moment Distribution due Unit Harmonic Moment at Pile Cap
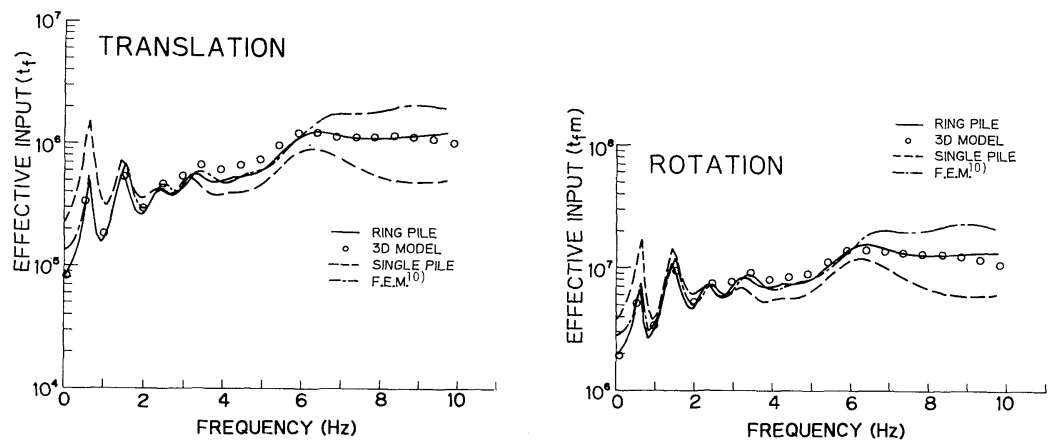

Fig.9 Effective Input (Transmitting Functions for Driving Forces)

frequency range since the effective input is defined by the product of the foundation impedance and the free field response. The analyses which account for the pile-soil-pile interaction give almost the same results in the significant frequency range. The small discrepancy between the DSP and the FEM approaches in the high frequency range is due to the accuracy of the discretization of the soils.

\section{CONCLUSION}

The authors presented a 3-D dynamic pile-soil-pile interaction from the DPS (beam analysis) method and its effective simplified method. From the numerical example the latter approximate method is verified to work well for seismic analysis of grouped pile foundations. The DPS solution is compared with the finite element solution.

From the engineering point of view, the pile head impepedance function and the associated driving force are investigated. The dynamic grouping effect is noted to vary depending on the driving frequency, 
indicating almost constant values less than 1 in the low frequency range while a greater values than 1 in the high frequency range. Remarks is also given, with special interest on pile grouping effect, on the load distribution to the respective piles from the pile cap. Once the pile head impedance and the associated driving force are obtained, the interaction analysis of soil-pile-superstructure is straight-forward from the dynamic substructure method with use of the superstructural natural modes (component mode method) ${ }^{5,10,11)}$.

\section{REFERENCES}

1) Tajimi, H. : Dynamic Analysis of a Structure Embedded in An Elastic Stratum, Procs. 4 th World Conf. Earthq. Eng., Chile, 1969.

2) Novak, M. : Dynamic Stiffness and Damping of Piles, Canadian Geotechnical Journal, Vol. 11, pp. 574 598, 1974.

3) Novak, M. and Nogami, T. : Soil-Pile Interaction in Horizontal Vibration, Earthquake Engineering and Structural Dynamics, Vol. 5, pp. 263 281, 1977 .

4) Novak, M. and Aboul-Ella, F. : Impedance Functions of Piles in Layered Media, J. Engrg. Mech. Div., ASCE, Vol. 104, pp. 643 661, 1978 .

5) Takemiya, H. and Yamada, Y. : Layered Soil-Pile-Structure Dynamic Interaction, Earthquake Engineering and Structural Dynamics, Vol. 9, pp. 437 457, 1981.

6) Wolf, J. P. and Von Arx, G. A. : Impedance Function of a Group of Vertical Piles, Procs., ASCE, Geotech. Eng. Div. Spec. Conf., Vol. 2, pp. 1024 1041, 1978.

7) Waas, G. and Hartmann, H. G. : Analysis of Pile Foundations Under Dynamic Loads, Procs. 6 th Int. Conf. Struct. Mech. Reactor Tech., Vol. K 5/2, France, 1981.

8) Nogami, T. : Dynamic Grouping Effect in Axial Responses of Grouped Piles, J. Geotech. Eng., ASCE, Vol. 109, No. 2, pp. 228 $\sim 243,1983$.

9) Ettouney, M. M., Brennan, J. A. and Forte, M. F. : Dynamic Behavior of Pile Groups, J. Geotech. Eng., ASCE, Vol. 109, No. 3, pp. 301 317, 1983.

10) Takamiya, H. : Ring Pile Analysis for Grouped Piles Subjected to Base Motion (to appear in Proc. Struc. Eng. /Erthq. Eng., JSCE, Vol.3, No. 1, pp. 195s 202s, April 1986).

11) Takemiya, H. and Yamada, Y. : Seismic Response Analysis of Multi-Span Continuous Bridge with High Piers on Deep Pile Foundations, Procs. Int. Conf. Soil Dynamic and Earthquake Engineering. Southamptong. Vol. 1.2, pp. 749 763, 1982.

(Received April 9 1985) 